\title{
Variogram-based method for contrast measurement
}

\author{
Luis Miguel Sanchez-Brea, ${ }^{*}$ Francisco Jose Torcal-Milla, and Eusebio Bernabeu \\ Department of Optics, Applied Optics Complutense Group, Universidad Complutense de Madrid, \\ Facultad de Ciencias Físicas, Ciudad Universitaria s.n., 28040 Madrid, Spain
}

${ }^{*}$ Corresponding author: sanchezbrea@fis.ucm.es

Received 9 January 2007; revised 5 April 2007; accepted 8 April 2007;

posted 9 April 2007 (Doc. ID 78790); published 6 July 2007

\begin{abstract}
We present a technique for determining the contrast of an intensity distribution in the presence of additive noise and other effects, such as undesired local amplitude or offset variations. The method is based on the variogram function. It just requires the measurement of the variogram at only four points and, as a consequence, it is very fast. The proposed technique is compared with other standard techniques, showing a reduction in the error of the contrast measurement. @ 2007 Optical Society of America

OCIS codes: $100.0100,070.6020,070.6760,100.2650$.
\end{abstract}

\section{Introduction}

Contrast measurement is a useful tool in several optics applications, such as optical metrology, image processing, etc. where sinusoidal fringes are obtained [1-4]. For example, a direct method for determining the modulation transfer function (MTF) of an optical system is measuring the contrast of several sinusoidal fringe patterns with different spatial frequencies imaged by the optical system under test. The definition of contrast is

$$
C=\frac{I_{\max }-I_{\min }}{I_{\max }+I_{\min }}
$$

where $I_{\max }$ and $I_{\min }$ are the maximum and minimum values of the signal, respectively. Let us consider that the signal can be modeled as a sinusoidal function:

$$
I(x)=B+A \sin \left(\frac{2 \pi x}{p}\right)+r(x),
$$

where $r(x)$ is a stochastic additive noise that responds to a Gaussian distribution with null mean value $\langle r(x)\rangle=0$, root mean square $\left\langle r(x)^{2}\right\rangle=\sigma^{2}$ and whose autocorrelation function is $\langle r(x) r(x+h)\rangle=\sigma^{2} \delta(h), \delta$ is

0003-6935/07/225027-07\$15.00/0

(C) 2007 Optical Society of America the Dirac-function $\delta$, and $\langle *\rangle$ represents averaging. When noise is null, the nominal contrast of the signal proposed in Eq. (2) is

$$
C_{\text {nom }}=\frac{A}{B}
$$

On the other hand, when the signal presents noise or background variations, it is not convenient to apply Eq. (1) directly for contrast measurement since the determination of $I_{\max }$ and $I_{\min }$ is very sensitive to noise. The contrast obtained using Eq. (1) gives

$$
C_{s t}=\frac{A+K \sigma}{B}
$$

where $K$ is a factor that depends on the number of data used, which normally ranges between 2 and 3 [5]. The relative error in the contrast measurement is

$$
e_{s t}=\left|\frac{C_{s t}-C_{n o m}}{C_{n o m}}\right|=K \frac{\sigma}{A},
$$

which is linear with $\sigma / A$. A better approach to determine the amplitude of the signal is the root mean square (rms) function, defined as [6]: 


$$
\mathrm{rms}=\left(\frac{\int[I(x)-\langle I\rangle]^{2} \mathrm{~d} x}{\int \mathrm{d} x}\right)^{1 / 2} .
$$

With this definition, all the data are considered for determining the amplitude of a signal, instead of just two data. The contrast gives

$$
C_{r m s}=\sqrt{2} \frac{\mathrm{rms}}{\langle I\rangle} .
$$

Using Eq. (6), the amplitude for a sinusoidal function without noise is then $A=\sqrt{2} \mathrm{rms}$. However, when an additive noise that responds to a Gaussian distribution noise defined previously in Eq. (2) is present, then $\mathrm{rms}^{2}=A^{2} / 2+\sigma^{2}$. The mean value of Eq. (2) is $\langle I\rangle=B$ and, as a consequence, the contrast gives

$$
C_{r m s}=\frac{\sqrt{A^{2}+2 \sigma^{2}}}{B} .
$$

This technique is also affected by the presence of an additive Gaussian noise. The relative error in the contrast estimation is

$$
e_{r m s}=\left|\frac{C_{r m s}-C_{n o m}}{C_{\text {nom }}}\right|=\sqrt{1+2\left(\frac{\sigma}{A}\right)^{2}}-1 .
$$

When noise is small compared with the amplitude of the signal, then $e_{r m s} \approx(\sigma / A)^{2}$, which is lower than with Eq. (5). On the other hand, when noise is much greater than the amplitude of the signal, the error in the contrast estimation presents a linear dependence, $e_{r m s} \approx \sqrt{2} \sigma / A$.

Another possibility to determine the contrast is to fit the experimental data to a sine function. However, experimentally a number of effects such as local variations of the period of the fringes, local variations of the amplitude or offset of the signal, normally appear, which avoids a correct fitting. Other robust techniques to evaluate the contrast of a fringe pattern have been proposed, such as the histogram, suggested by Lai and von Bally [7]. Nevertheless, the algorithm proposed in that work does not match the definition of contrast given by Eq. (1), even when no noise is present. A better technique for contrast measurement in presence of additive noise and with patterns composed of nonstraight fringes has been developed [8]. It is based on fitting the histogram of the fringe pattern to the histogram of a model function that depends on several parameters. The technique has been proven to obtain good results for contrast estimation even with the presence of high levels of noise. However, the computation algorithm is quite slow as it requires the fitting of the histogram to a function with several parameters.

In this work, a completely different approach is applied for estimating the contrast. Using the variogram function $\gamma(h)$, see Section 2, we demonstrate that the difference between the maximum and minimum values of the variogram is equal to the square of the amplitude of the signal, even when an additive noise is present. Since the variogram is obtained as an average process, noise affects very little to the contrast estimation. An expression for the relative error in the contrast determination is obtained showing that it depends only on the signal-to-noise ratio and on the number of data. Numerical simulations with different modifications on the signal are performed that corroborates the robustness and validity of the technique. Finally, as an example, the contrast of signals obtained at a certain distance from a diffraction grating when it is illuminated with a monochromatic collimated light beam are obtained. This corresponds to the well-known Talbot effect.

\section{Theoretical Analysis}

To determine the amplitude of a sinusoidal intensity distribution, let us consider the semivariogram function $[9,10]$, which is defined as

$$
\gamma(h)=\frac{1}{2}\left\langle[I(x+h)-I(x)]^{2}\right\rangle,
$$

where $\langle *\rangle$ means averaging with respect to $x$. For the sinusoidal signal described in Eq. (2), the semivariogram results in

$$
\gamma(h)=A^{2} \sin ^{2}\left(\frac{\pi h}{p}\right)+\sigma^{2}[1-\delta(h)] .
$$

The maximum value of the variogram is obtained at $p / 2, \gamma(p / 2)=A^{2}+\sigma^{2}$. The variogram at the origin is, according to Eq. (11), $\gamma(0)=0$. However, the semivariogram is discontinuous at the origin and the extrapolated value is $\hat{\gamma}(0)=\sigma^{2}$, [11], where $\hat{\gamma}$ means that the value has been obtained using an extrapolation. Several techniques have been proposed for determining the extrapolate value $\hat{\gamma}(0)[9-12]$. The fastest way is to use the value of the semivariogram at the nearest point $\hat{\gamma}(0) \approx \gamma(\Delta x)$. In most practical situations, the variogram presents a quadratic dependence near the origin. As a result, a better and still fast procedure is to consider a quadratic extrapolation with the first three points of the semivariogram,

$$
\hat{\gamma}(0) \approx 3[\gamma(\Delta x)-\gamma(2 \Delta x)]+\gamma(3 \Delta x) .
$$

Considering Eq. (11), the amplitude of the signal can be measured with

$$
A=\sqrt{\gamma(p / 2)-\hat{\gamma}(0)},
$$


and the contrast is, as a consequence,

$$
C_{\gamma}=\frac{\sqrt{\gamma(p / 2)-\hat{\gamma}(0)}}{\langle I\rangle} .
$$

Using this definition, the relative error in the contrast estimation using the semivariogram is

$$
e_{\gamma} \approx\left|\frac{C_{\gamma}-C_{n o m}}{C_{\gamma}}\right|=0 .
$$

Another effect that should be considered when determining the relative error in the contrast estimation is the random fluctuations of the contrast due to a sampling at discrete locations.

In the first two techniques, Eqs. (4) and (8), the random fluctuations are normally much smaller than the variations from the nominal contrast given in Eq. (3). However this is not the case for the variogrambased technique since the relative error given by Eq. (15) is null. For a regularly sampled signal, the variogram is computed using

$$
\gamma(n \Delta x)=\frac{1}{2(N-n)} \sum_{i=1}^{N-n}\left(I_{i+n}-I_{i}\right)^{2}
$$

where $N$ is the number of data, $I_{i}=I\left(x_{0}+i \Delta x\right)$ is the measured value at $x_{i}=x_{0}+i \Delta x, i=1,2, \ldots, N$, and $\Delta x$ is the distance between two adjacent points of the discrete variogram. The variogram estimation is affected by the random variations of $I_{i}$. Performing a simple error propagation of Eq. (14), [13], then the normalized uncertainty in the contrast estimation of the variogram-based technique gives

$$
e_{\gamma, s t} \approx \frac{1}{\sqrt{N}} \frac{\sigma}{A}+\sqrt{\frac{2}{N}}\left(\frac{\sigma}{A}\right)^{2}
$$

which depends on the number of data $N$. For signals obtained with linear or bidimensional CCD cameras, $N$ is quite large, and this normalized uncertainty is much lower than the error obtained with the other techniques presented, as shown in Section 3.

The number of sums required to determine the contrast using Eq. (14) is approximately $3 N$. As a consequence, this algorithm is much faster that the "histogram fitting" method proposed in [8] where the optimization required more than $1 \mathrm{~min}$ for obtaining the result. With the proposed algorithm, the computing time (Pentium IV, $2000 \mathrm{~Hz}$ ) was lower than $0.5 \mathrm{~ms}$ for $N=1000$. Therefore, this technique can be applied for real-time processing. The computation speed can also be improved using a digital signal processor (DSP).

\section{Numerical Simulations}

\section{A. Additive Gaussian Noise}

To check the validity of the proposed technique, we have applied it to numerous signals with different

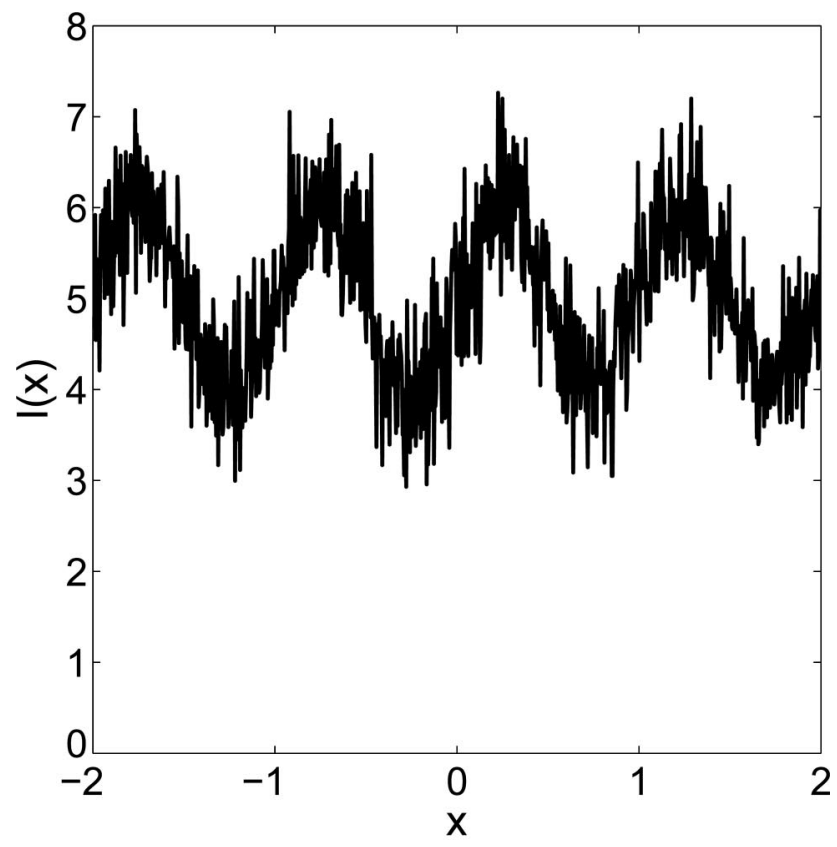

(a)

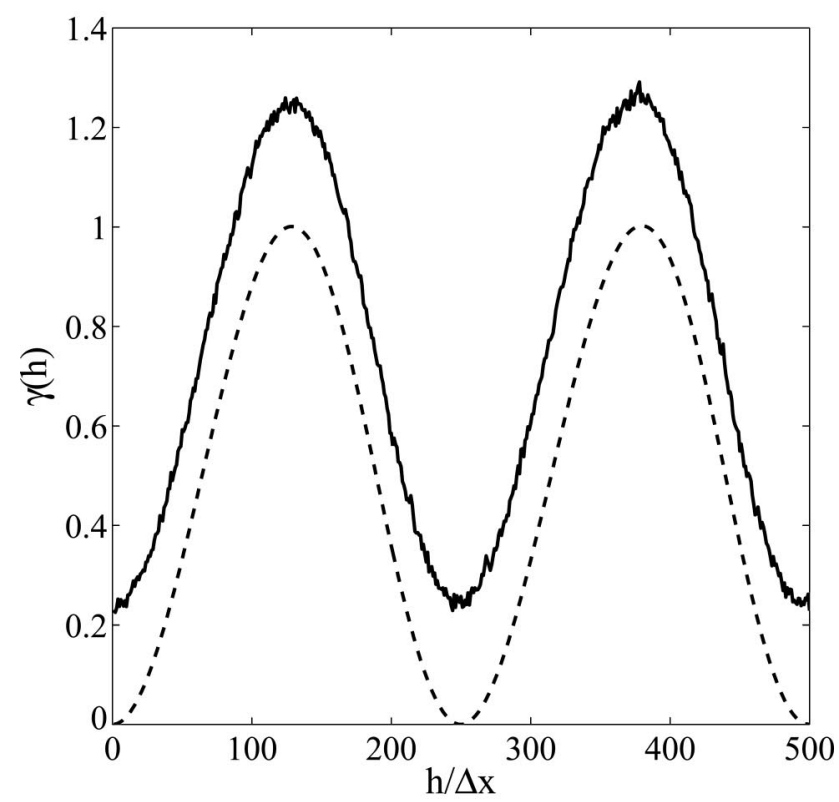

(b)

Fig. 1. (a) Signal $I(x)=5+\sin (2 \pi x)+r(x)$, where $r(x)$ represent an additive Gaussian noise with standard deviation $\sigma=0.5$. (b) Variogram for this signal when $\sigma=0$, dashed curve, and $\sigma=$ 0.5 , solid curve.

parameters and adverse effects. As an example, in Fig. 1, we show the effect of a Gaussian additive noise on the contrast estimation. We have used the following function $I(x)=5+\sin (2 \pi x)+r(x)$, which has been sampled on the interval $x \in(-2,2)$ with 500 regularly distributed observations. The characteristics of $r(x)$ are those presented in Eq. (2). The contrast has been estimated for several values of $\sigma$. In Figs. 1 (a) and 1(b), this function is shown for $\sigma=0.5$, as well as the semivariograms for $\sigma=0$ and $\sigma=0.5$. 


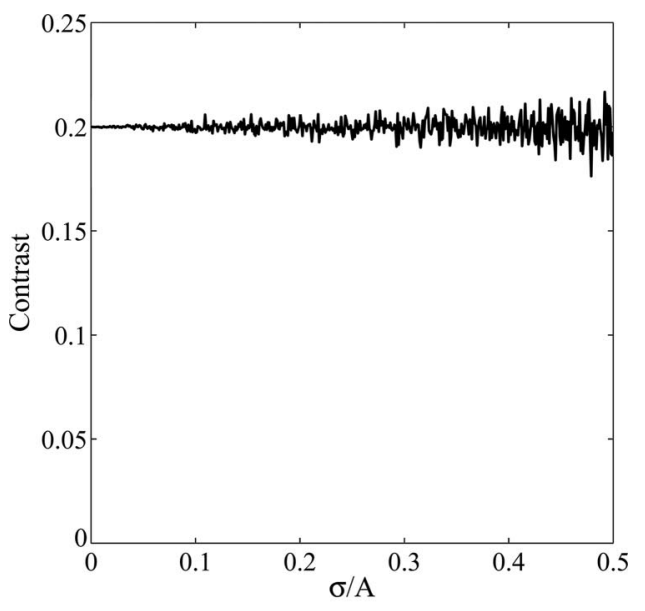

(a)

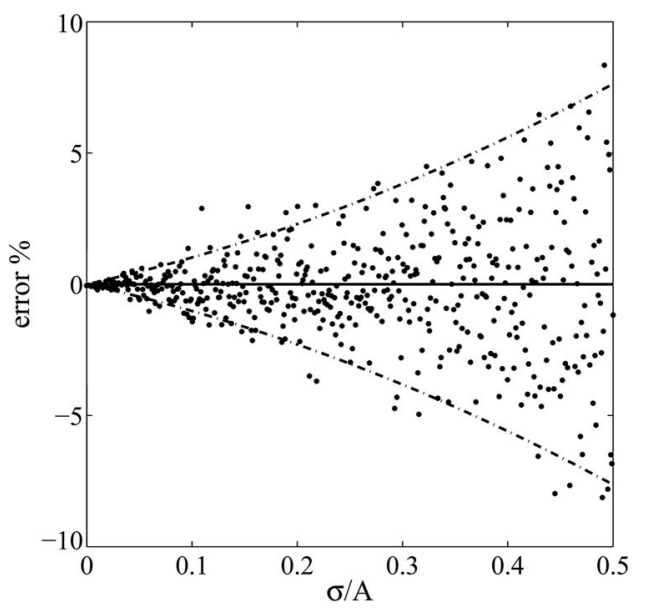

(b)

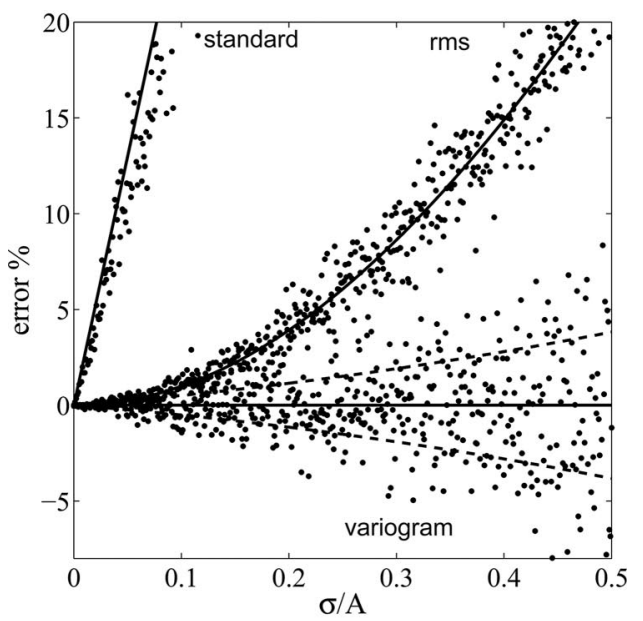

(c)

Fig. 2. (a) Contrast estimated with the variogram-based technique, Eq. (14), for the signal of Fig. 1, for different values of noise. (b) Relative error in the contrast estimation: error of the simulation, circles, error estimated with Eq. (15), solid line, and $\pm 2 e_{\gamma, s t}$, given in Eq. (17), dashed-dotted. (c) Comparison of the three techniques presented in the work (standard technique, rms technique, and variogram technique). In all the cases, circles represent the relative error using the technique; thick curves represent the average error given by Eqs. (5), (9), and (15), respectively. Dasheddotted curves for the variogram-based technique represent $\pm e_{\gamma, s}$.
While the function $f$ presents a strong fluctuation, the semivariogram is quite smooth, since it is obtained as an averaging. In Fig. 2(a), the contrast obtained using the variogram-based technique is shown for different values of $\sigma$, and in Fig. 2(b), the error in the estimation is compared with Eq. (15). A quadratic fit to the numerical data has been included, which corroborates the validity of Eq. (15) for predicting the error in the contrast estimation. Finally, in Fig. 2(c), the three techniques (direct definition, rms, and variogram) have been compared for different noise levels. For very low noise, the rms technique and the variogrambased technique for contrast estimation present a similar behavior. However, for high noise levels, the proposed variogram-based technique is much better.

\section{B. Offset Fluctuations}

We have also analyzed the validity of the variogrambased method when other effects on the signal appear as, for example, when the signal presents offset fluctuations. There are many cases where these local contrast variations provide us with information about fluctuations of the parameter to measure. However, there are also many situations where they are due undesired effects, such as dust or a nonuniform illumination. Then, an average of the contrast is required. This is the case of the experimental example presented in Section 4, where local inhomogeneities in the light source or the gratings produce undesired fluctuations in the offset or amplitude of the signals.

As an example, we have simulated the following offset fluctuations: $I(x)=[5+k \sin (\pi x)]+\sin (8 \pi x)$ in order to show that the variogram based technique is valid to determine the average contrast value. This function has been sampled 1000 points between $x \in(-2,2)$. The first sine function acts as a slow variation function that avoids a correct average contrast estimation using standard techniques. In Fig. $3(\mathrm{a})$, the function $I(x)$ for $k=0.5$ is shown, and in Fig. $3(\mathrm{~b})$, the relative error in the contrast estimation is shown for several values of $k$ and for the three techniques. The variogram-based technique for contrast estimation is not very affected for this fluctuation, while the other techniques present a worse behavior.

\section{Fluctuations in the Amplitude}

Local variations in the amplitude may also affect to the contrast estimation. Several simulations have been performed that show that the variogram technique for contrast estimation is better than the other standard techniques. As an example, in Fig. 4(a), the signal $I(x)=10+[1+a \cos (\pi x / 2)] \sin (4 \pi x)$ is shown for $a=0.5$, and in Fig. 4(b), the relative error in the contrast estimation is shown for different values of $a$. We can also see that the relative error is lower for the variogram technique than for the other standard techniques.

\section{Experimental Results: Application to Talbot Effect}

To experimentally show the advantage of the variogram-based technique with respect to other techniques for contrast estimation, we have measured the 


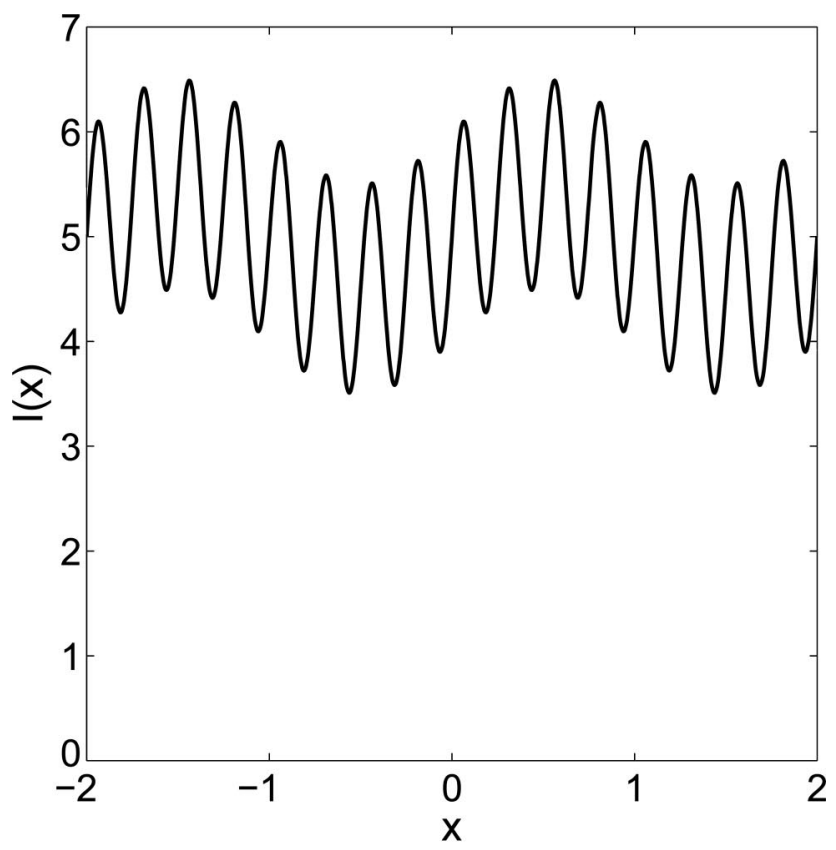

(a)

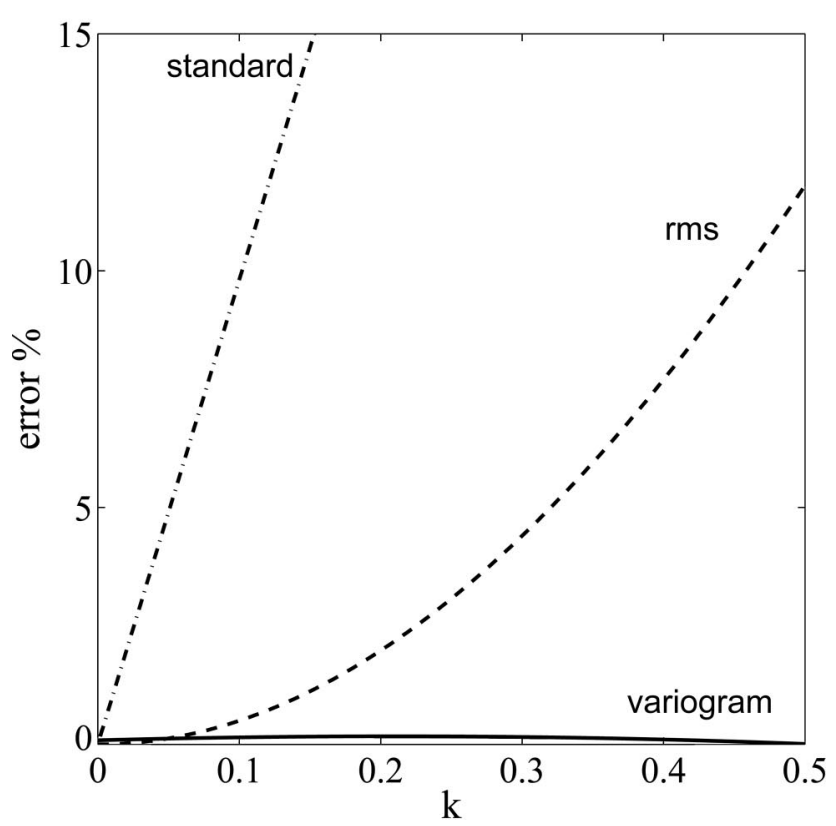

(b)

Fig. 3. (a) Signal $I(x)=5+\sin (8 \pi x)+k \sin (\pi x)$ sampled 1000 points between $x \in(-2,2)$ for $k=0.5$. (b) Relative error in the contrast estimation in terms of $k$ : Direct definition of contrast, Eq. (1) dashed-dotted curve; rms technique, Eq. (7), dashed curve; and variogram technique, Eq. (14), solid curve.

contrast for the case of Talbot effect [14]. For this, we have used a diffraction grating made of chrome on a glass substrate with a period of $100 \mu \mathrm{m}$. In Fig. 5(a), the experimental intensity distribution captured with a complementary metal-oxide semiconductor (CMOS) camera is shown in terms of the distance $z$ between the diffraction grating and the observation plane. It appears to be a self-imaging process. The

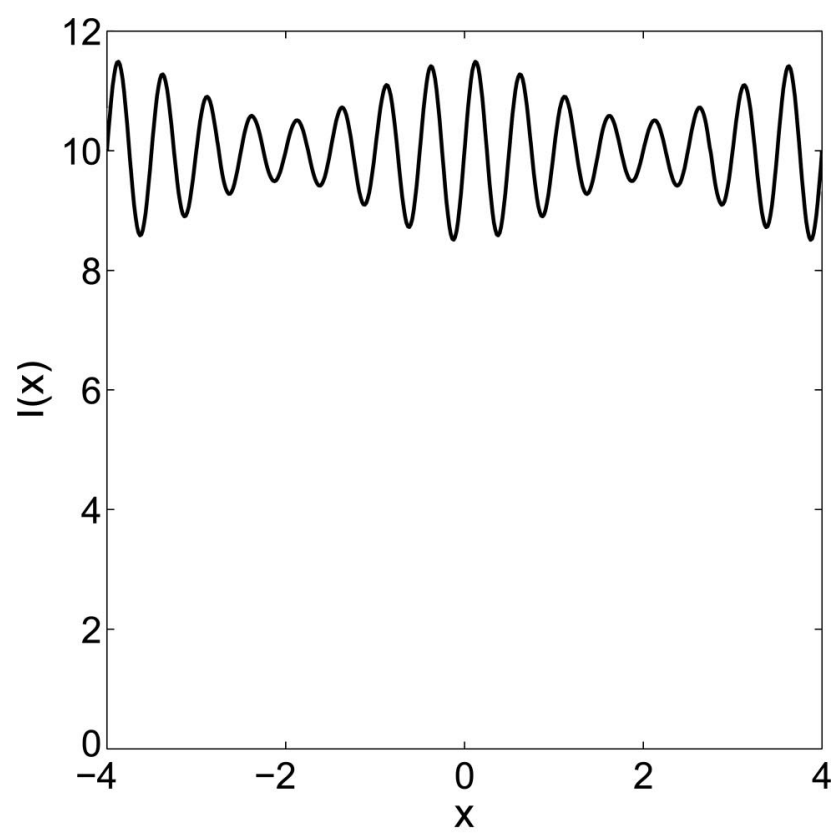

(a)

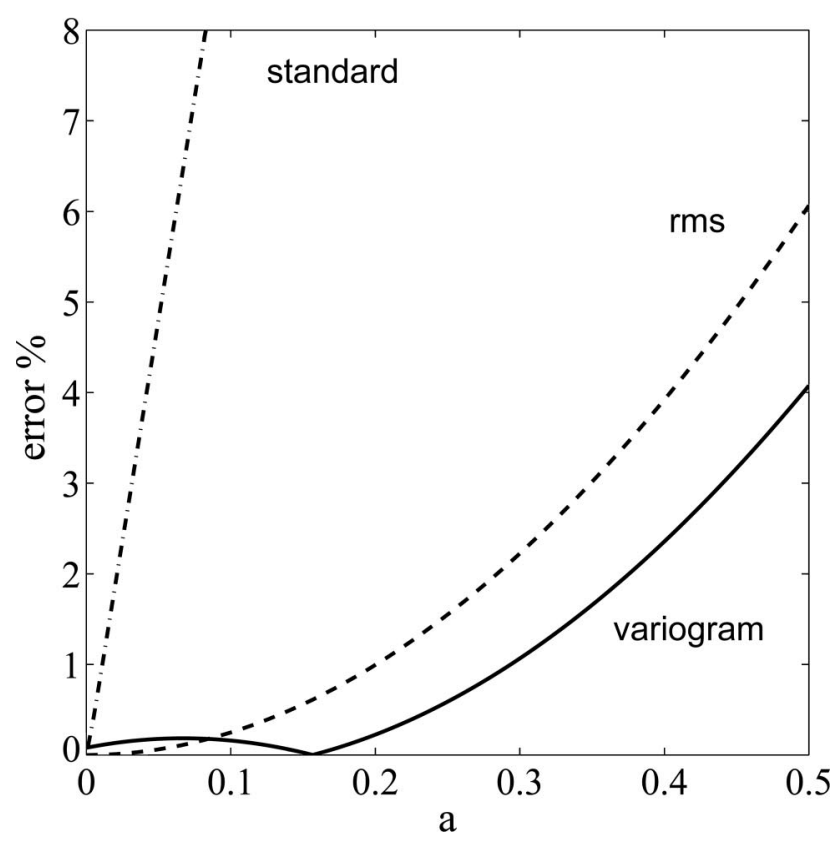

(b)

Fig. 4. (a) Signal $I(x)=10+[1+a \cos (\pi x / 2)] \sin (4 \pi x)$ sampled 1000 points between $x \in(-2,2)$ for $a=0.5$. (b) Relative error in the contrast estimation in terms of $a$ : Direct definition of contrast, Eq. (1) dashed-dotted; rms technique, Eq. (7) dash and variogram technique, Eq. (14) solid.

grating is reproduced at regular distances, known as Talbot planes. In Figs. 5(b) and 5(c), the fringes obtained at this Talbot distance, which present a high contrast the fringes for a transition zone (low contrast fringes), are shown. In Fig. 6, the contrast obtained at different distances $z$ from the grating to the observation plane are determined using the techniques presented in this work. As observed, the variogram 


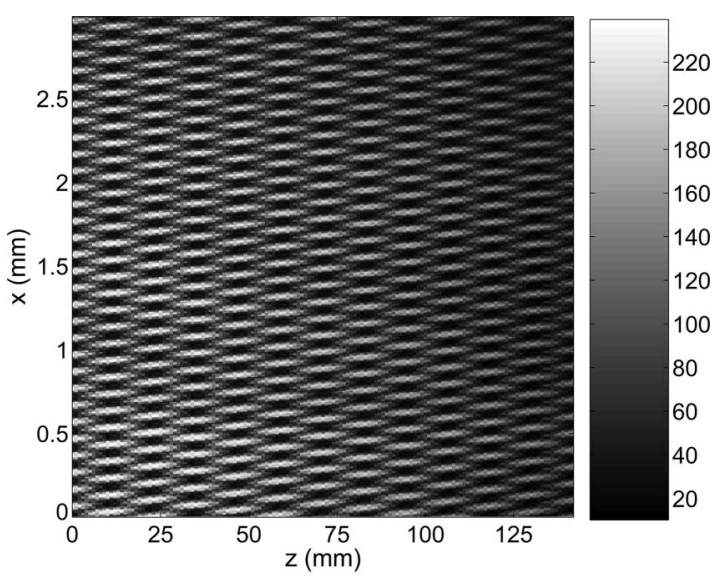

(a)

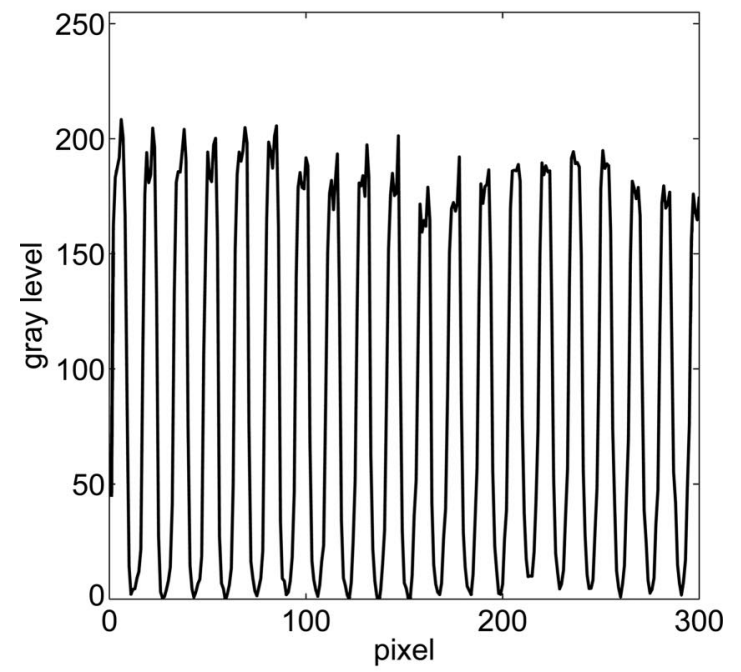

(b)

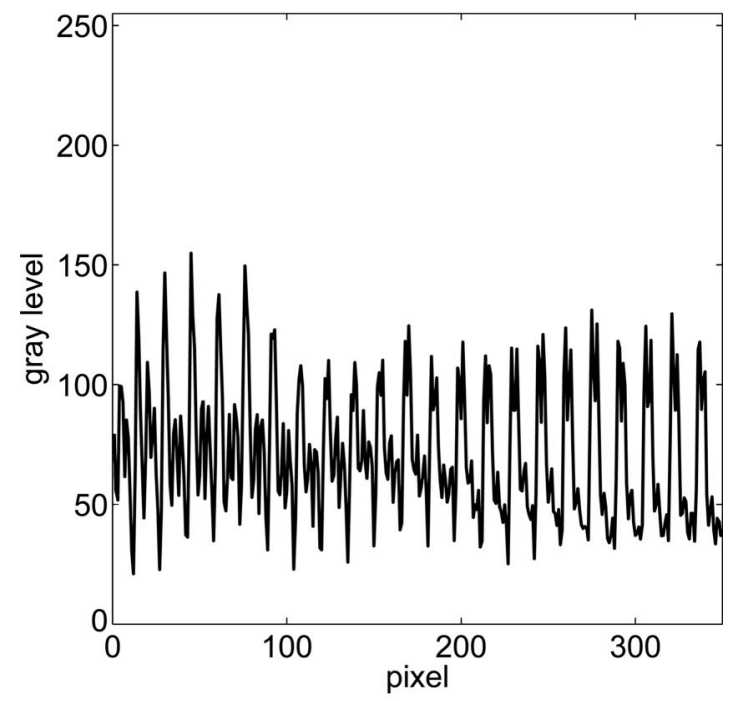

(c)

Fig. 5. (a) Experimental intensity obtained after a diffraction grating (period $100 \mu \mathrm{m}$ ) when it is illuminated with a monochromatic plane wave (wavelength $670 \mathrm{~nm}$ ). $Z$ is the distance between the grating and a CMOS camera. Talbot planes are observed. (b) Fringes obtained for a position of high contrast and (c) fringes for a transition zone where contrast is low.

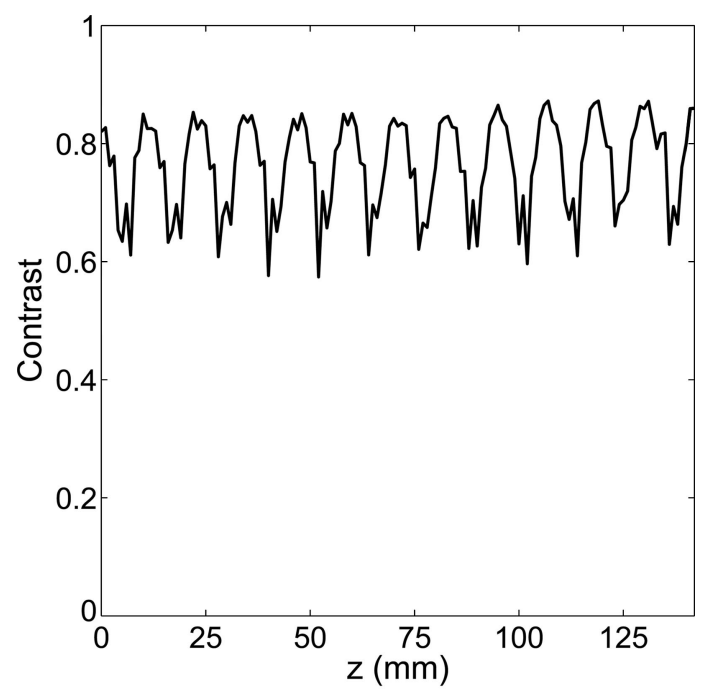

(a)

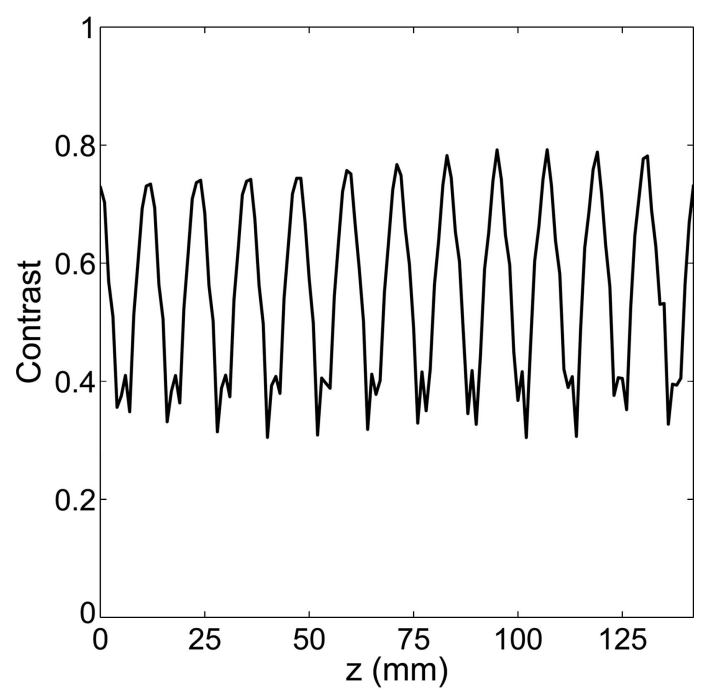

(b)

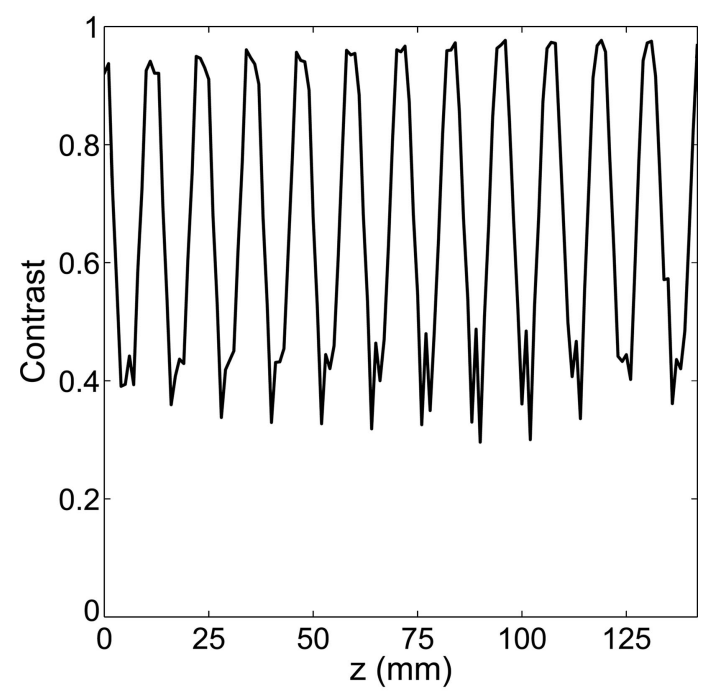

(c)

Fig. 6. Contrast obtained with the different techniques presented; (a) direct definition of contrast, Eq. (1). (b) Rms technique, Eq. (7), and (c) variogram technique, Eq. (14). 
technique for contrast estimation produces a higher contrast difference, which is in accordance with the theoretical results.

\section{Conclusions}

In this work, a variogram-based technique for robust contrast measurement is presented. With this technique, the contrast is not obtained considering only the maxima and minima of the signal, but all the data are used. As a consequence, this technique for contrast measurement is quite robust. In addition, an expression for the error committed in the contrast evaluation is given. The technique has been proven using simulations with known additive Gaussian noise. Also, other modifications to the sinusoidal signals have been tested such as including amplitude or background variations. The value of the contrast and the error have been compared with the theoretical expressions obtained, both being in well accordance. Also an experimental example showing the validity of the variogram-based method is presented, as it improves the results obtained with other standard techniques.

The authors thank Tomás Morlanes for his valuable ideas. This work has been supported by the "Codificación óptica de la posición a escala nanométrica: Nuevas tecnologías y dispositivos ópticos" project (DPI2005-02860) of the Ministerio de Educación y Ciencia of Spain and the "Tecnologías en ecología, alta precisión y productividad, multifuncionalidad, y tecnologías de la información y comunicaciones en Máquina Herramienta" CENIT project of the Ministerio de Industria, turismo y comercio. Sanchez-Brea is currently contracted by the Univer- sidad Complutense de Madrid under the "Ramón y Cajal" research program of the Ministerio de Educación y Ciencia of Spain.

\section{References}

1. G. C. Holst, CCD Arrays, Cameras, and Displays (Society for Photo-Optical Instrumentation Engineers, 1996).

2. P. Hariharan, Optical Interferometry (Academic, 1989).

3. J. W. Goodman, Introduction to Fourier Optics (McGraw-Hill, 1996).

4. W. K. Pratt, Digital Image Processing (Wiley, 1978).

5. International Standardization Organization, Guide to the Expression of the Uncertainty in Measurement, Geneva (ISO, 1995).

6. E. W. Weisstein, "Root-mean-square," From MathWorld-A Wolfram Web Resource. http://mathworld.wolfram.com/RootMean-Square.html.

7. S. Lai and G. Von Bally, "Fringe contrast evaluation by means of histograms," in OPTIKA '98: 5th Congress on Modern Optics, G. Ákos, G. Lupkovics, and P. András, eds. Proc. SPIE 3573, 384-387 (1998).

8. L. M. Sanchez-Brea, J. A. Quiroga, A. Garcia-Botella, and E. Bernabeu, "Histogram-based method for contrast measurement," Appl. Opt. 39, 4098-4106 (2000).

9. R. Christiensen, Linear Models for Multivariate, Time Series, and Spatial Data (Springer-Verlag, 1985).

10. N. A. Cressie, Statistics for Spatial Data (Wiley, 1991).

11. L. M. Sanchez-Brea and E. Bernabeu, "On the standard deviation in CCD cameras: a variogram-based technique for nonuniform images," J. Electron. Imaging 11, 121-126 (2002).

12. L. M. Sanchez-Brea and E. Bernabeu, "Estimation of the standard deviation in three-dimensional microscopy by spatial statistics," J. Microsc. 218, 193-197 (2005).

13. P. Bevington, Data Reduction and Error Analysis for the Physical Sciences (McGraw-Hill, 1969).

14. E. Keren and O. Kafri, "Diffraction effects in moire deflectometry,” J. Opt. Soc. Am. A 2, 111-120 (1985). 